\title{
Intrinsic kinetics of biofilms formed under turbulent flow and low substrate concentrations
}

\author{
M.J. Vieira, L.F. Melo
}

\begin{abstract}
Reactor operating conditions strongly affect the behaviour of biofilm systems, namely their stability and the substrate removal. In this paper, the penetration of substrate and the activity of biofilms formed by Pseudomonas fluorescens under turbulent flow and low substrate concentrations, are studied. A first order diffusion-reaction model was applied to results of biofilm accumulation in steady and non-steady-state. The substrate consumption rate of the biofilm was calculated based on the on-line determination of the biofilm accumulated on the surface. This approach is important when the residence time or the substrate concentration on the reactor is very low. Also, the mass transfer of substrate inside the biofilm was measured for every case under study and introduced in the model.

The fraction of biofilm penetrated by the substrate depends on the velocity of the fluid that contacts the biological matrix: contrary to biofilms formed at higher velocities, lower velocities give raise to non completely penetrated biofilms. This fact seems to be associated to the biofilm internal structure in terms of biomass density and compactness of the matrix. They remove more substrate per reactor volume, but are less resistant from an hydrodynamic point of view. In conclusion, biofilms formed at higher velocities in turbulent flow allow a more stable reactor operation.
\end{abstract}

\section{List of symbols}

$C_{\mathrm{b}} \quad\left(\mathrm{kg}_{\text {substrate }} / \mathrm{m}^{3}\right)$

$C_{\mathrm{f}} \quad\left(\mathrm{kg}_{\text {substrate }} / \mathrm{m}^{3}\right.$ biofilm $)$

$C_{\mathrm{f}}^{*} \quad\left(C_{\mathrm{f}}^{*}=C_{\mathrm{f}} / C_{\mathrm{s}}\right)$

$C_{\mathrm{s}} \quad\left(\mathrm{kg}_{\text {substrate }} / \mathrm{m}^{3}\right.$ biofilm $)$ bulk substrate concentration concentration of limiting substrate at any point in the biofilm

dimensionless concentration in the position $\mathrm{x}^{\star}$ of the biofilm concentration in the liquidbiofilm interface
Received: 5 May 1998

M.J. Vieira, L.F. Melo

IBQF/Center of Biological Engineering

University of Minho, 4710 Braga, Portugal

Correspondence to: L.F. Melo

The financial support of Programme PRAXIS XXI, through Contract no. 2.1/BIO/37/94 and of Programme INTERREG, through Contract no. 01/REG II/6/96 is gratefully acknowledged.

$\begin{array}{ll}D_{\mathrm{f}} & \left(\mathrm{m}^{2} / \mathrm{s}\right) \\ J_{\mathrm{p}} & \left(\mathrm{kg}_{\text {biofilm }} / \mathrm{m}^{2} \mathrm{~s}\right) \\ \mathrm{k}_{1 \mathrm{f}} & \left(\mathrm{s}^{-1}\right) \\ k_{\mathrm{b}} & (\mathrm{m} / \mathrm{s}) \\ k_{\mathrm{m}} & (\mathrm{m} / \mathrm{s}) \\ \mathrm{k}_{\mathrm{s}} & \left(\mathrm{kg}_{\text {substrate }} / \mathrm{m}^{3}\right) \\ L_{\mathrm{f}} & (\mathrm{m}) \\ m_{\mathrm{f}} & \left(\mathrm{kg}_{\text {biofilm }} / \mathrm{m}^{2}{ }_{\text {biofilm }}\right) \\ r_{\mathrm{fa}} & \left(\mathrm{kg}_{\text {substrate }} / \mathrm{m}^{2} \mathrm{~s}\right) \\ r_{\mathrm{fmax}} & \left(\mathrm{kg}_{\text {substrate }} / \mathrm{m}^{3}{ }_{\text {biofilm }} \mathrm{s}\right) \\ x^{*} & \left(x^{*}={ }_{x} / L_{\mathrm{f}}\right) \\ Y_{\mathrm{X} / \mathrm{S}} & \left(\mathrm{kg}_{\text {biomass }} / \mathrm{kg}_{\text {substrate }}\right)\end{array}$

average "effective diffusivity" biofilm production flux the first order reaction rate constant mass transfer coefficient in the biofilm external mass transport co efficient saturation constant thickness of the biofilm. mass of biofilm per unit area overall rate of substrate con sumption

) maximum rate of substrate

utilization

dimensionless distance

within the biofilm

biomass yield

\section{Greek symbols}

$\phi$

biofilm constant (Thiele modulus)

effectiveness factor

\section{$\mu_{\mathrm{p}} \quad$ (kgiofilm produced}

$/ \mathrm{kg}_{\text {biofilm on the surface }} \mathrm{s}$ ) biofilm specific production rate

$\rho_{\mathrm{f}} \quad\left(\right.$ dry $\mathrm{kg}_{\text {biofilm }} / \mathrm{m}^{3}$ biofilm $)$ dry density of the biofilm (dry mass per unit volume)

\section{1}

\section{Introduction}

Nowadays, the reactors used for wastewater treatment and, in particular for water treatment where low substrate concentrations prevail operate under high fluid velocities, as in the case of biofilm air-lift reactors for water nitrification and fluidized bed reactors for nitrogen and carbon removal. In these systems, as well as when biofouling occurs in heat exchangers and in drinking water pipes, biofilm growth is controlled by the shear forces applied on the biofilm surface by the fluid flowing over it [16]. The operating conditions will determine the structure of the matrix and affect the internal substrate transport, bacterial concentration and reaction rate.

The overall "observable" or apparent reaction rate in a biofilm system comprises the transport of the reactants outside and inside the biological matrix and the biochemical reaction involving the immobilized microorganisms. The diffusion-reaction models developed for 
heterogeneous catalysis may be applied to describe the kinetics of biofilms, since in both cases the reactants must be transported through a porous structure before being consumed.

Several authors have already used similar models to describe the phenomena inside biofilms $[4,6,10]$, but they assumed that the substrates were transported through the biofilm by molecular diffusion. There is some evidence that convection may be a significant mechanism for substrate mass transport inside microbial films, as reported values for the diffusivity inside biofilms are sometimes higher than in pure water [7, 9]. Also, Stoodley et al. [14] and Lewandowski et al. [7] using confocal laser microscopy to study biofilms formed in contact with water at very low velocity, observed liquid flow within the biofilm channels and concluded that this liquid flow was related to the physical structure of the biofilm. Furthermore, the structure of a biofilm is dependent on the conditions under which it is formed, namely substrate loading rate, shear stress, bulk liquid velocity, type of reactor and microorganism $[3,12,16]$, which in turn affect the mass transfer inside the biofilm. Moreover, biofilms are reported to have different structural layers $[12,18]$ of different densities or in some cases, probably for low velocities, an heterogeneous structure consisting of cell clusters separated by channels can arise [14]. Therefore, it is not possible to find "unique" values for the diffusivity of a given substrate within biofilms, even when they are formed by the same microbial species. If an average mass transfer coefficient, comprising all the mass transport mechanisms in the biofilm, and the substrate consumption rate, are experimentally determined for each case under study, the diffusion-reaction model can be used to interpret the biofilm kinetics in a more reliable way, and to predict the effectiveness factors related to substrate consumption by the microbial aggregate.

In this paper a diffusion-reaction model (first order reaction with external mass transfer) is applied to steadystate and non steady-state biofilms formed under different hydrodynamic conditions, in turbulent flow and low organic substrate concentration.

A steady-state biofilm is one for which the biomass per unit surface area is constant with time and the rate of substrate consumption in the biofilm is also constant with respect to time [13]. At steady-state, the increase of biomass resulting from substrate consumption is balanced by the detachment processes (erosion) due to the shear effects caused by the fluid moving over the biofilm. In the present study, the sloughing off of large portions of the biofilm caused by internal or external phenomena producing abrupt changes in the thickness and in the consumption of nutrients by the biofilm is not considered, since it is a fortuituous process that was not significant during the experiments reported below.

The detachment processes are limited to the surface of the biofilm and occur from the beginning of biofilm formation, increasing as the amount of the biofilm on the surface increases [1]. For that reason, as far as the rate of detachment is lower than the rate of biofilm production, the amount of biofilm will increase, even if a constant rate of substrate consumption is observed. This period will be considered the non-steady period.

As shown in a previous paper [9] transport and adhesion of new cells to the surface are not relevant processes in the overall biofilm development rate, after the initial period of inoculation. Therefore, the biological growth of the biofilm can be expressed by a variable called the "biofilm production flux", $J_{\mathrm{p}}$, that involves all the phenomena that contribute in a positive way to the formation of the microbial layer, namely the transport of nutrients to the surface, followed by cell growth, reproduction and production of extracellular polymeric substances (EPS). These phenomena are competitive with, and simultaneous to detachment. The "biofilm production flux" is considered to be constant throughout the formation of the biological deposit, while the detachment rate increases with the amount of deposit present on the surface [9]. $J_{p}$ will be modelled below according to the diffusion-reaction model described by Harremöes [6].

\section{2}

\section{Model development}

\section{1}

\section{Rate of biofilm production}

Let $m_{\mathrm{f}}$ be the mass of biofilm per unit area $\left(\mathrm{kg}_{\text {biofilm }} /\right.$ $\mathrm{m}^{2}$ biofilm). The "biofilm production flux" in mass units $\left(\mathrm{kg}_{\text {biofilm }} / \mathrm{m}^{2} \mathrm{~s}\right), J_{\mathrm{p}}$, can be obtained from the "biofilm accumulation curves" ( $m_{\mathrm{f}}$ versus time) by calculating the slope at $t=0$ :

$J_{\mathrm{p}}=\left(\frac{\mathrm{d} m_{\mathrm{f}}}{\mathrm{d} t}\right)_{t=0}$

\section{2}

\section{Overall substrate consumption rate by the biofilm}

The overall rate of substrate consumption, $r_{\mathrm{fa}}\left(\mathrm{kg}_{\text {substrate }} /\right.$ $\mathrm{m}^{2} \mathrm{~s}$ ), can be calculated by the following equation, based on the rate of biofilm production rate:

$r_{\mathrm{fa}}=\frac{J_{\mathrm{p}}}{Y_{\mathrm{X} / \mathrm{s}}}$,

where $Y_{\mathrm{X} / \mathrm{S}}$ is the biomass yield, calculated as the ratio between the mass of biomass (cells and polymers) formed and the mass of nutrients consumed. It was considered that the biomass yield in the biofilm is equal to that in suspension [17].

\section{3}

\section{Kinetics of substrate consumption}

The substrate consumption at any point in the biofilm per unit volume of the attached layer is described by the Monod relationship:

$r_{\mathrm{f}}=r_{\mathrm{fmax}} \frac{C_{\mathrm{f}}}{\mathrm{k}_{\mathrm{s}}+C_{\mathrm{f}}}$

where $C_{\mathrm{f}}$ is the concentration of limiting substrate at any point in the biofilm, $\mathrm{k}_{\mathrm{s}}\left(\mathrm{kg}_{\text {substrate }} / \mathrm{m}^{3}\right)$ is the saturation constant, and $r_{\text {fmax }}$ is the maximum rate of substrate uti- 
lization $\left(\mathrm{kg}_{\text {substrate }} / \mathrm{m}^{3}\right.$ biofilm $\left.\mathrm{s}\right)$. The equation considers two extreme situations: zero order when the substrate concentration is high enough $\left(C_{\mathrm{f}}>>\mathrm{k}_{\mathrm{s}}\right)$ and first order for small concentrations of substrate.

The steady-state mass balance on the limiting substrate at any point of the biofilm is described by the equation:

$D_{\mathrm{f}} \frac{\mathrm{d}^{2} C_{\mathrm{f}}}{\mathrm{d} x^{2}}=r_{\mathrm{fmax}} \frac{C_{\mathrm{f}}}{\mathrm{k}_{\mathrm{s}}+C_{\mathrm{f}}}$,

where $D_{\mathrm{f}}$ is an average "effective diffusivity" that may include all types of mass transfer phenomena.

It was assumed that the kinetics of substrate consumption inside the biofilm is of first order in relation to the substrate concentration (supposing that the value of $\mathrm{k}_{\mathrm{s}}$ of Pseudomonas fluorescens is much higher than the concentrations of substrate in the biofilm). Thus, the differential equation to be solved is:

$D_{\mathrm{f}} \frac{\mathrm{d}^{2} C_{\mathrm{f}}}{\mathrm{d} x^{2}}=\mathrm{k}_{1 \mathrm{f}} C_{\mathrm{f}}$

where $\mathrm{k}_{1 \mathrm{f}}\left(\mathrm{s}^{-1}\right)$ is the first order reaction rate constant $\left(\mathrm{s}^{-1}\right)$, equal to $r_{\mathrm{fmax}} / \mathrm{k}_{\mathrm{s}}$. In dimensionless terms this equation becomes:

$\frac{\mathrm{d}^{2} C_{\mathrm{f}}^{*}}{\mathrm{~d} x^{* 2}}=\frac{\mathrm{k}_{1 \mathrm{f}} L_{\mathrm{f}}^{2}}{D_{\mathrm{f}}} C_{\mathrm{f}}^{*}=\phi^{2} C_{\mathrm{f}}^{*}$,

defining $C_{\mathrm{f}}^{*}$ as the dimensionless concentration in the position $x^{*}$ of the biofilm $\left(C_{\mathrm{f}}^{*}=C \mathrm{f} / C \mathrm{~s}\right.$ where $C_{\mathrm{s}}$ is the concentration in the liquid-biofilm interface), $x^{*}$ as the dimensionless distance within the biofilm $\left(x^{*}=x / L_{\mathrm{f}}\right)$, and $L_{\mathrm{f}}$ as the thickness of the biofilm. $\phi$ is the biofilm constant (Thiele modulus) and is defined as [6]:

$\phi=\sqrt{\frac{\mathrm{k}_{\mathrm{lf}} L_{\mathrm{f}}^{2}}{D_{\mathrm{f}}}}$,

The solution of Eq. (6) is the concentration profile in the biofilm:

$C_{\mathrm{f}}^{*}=\frac{\cosh \left[\phi\left(1-x^{*}\right)\right]}{\cosh \phi}$.

The effect of the internal diffusion resistance on the overall "observable" reaction rate is estimated by an effectiveness factor, defined as:

$\eta=\frac{\tan h \phi}{\phi}$,

which expresses the ratio between the substrate consumption by the biofilm and the substrate consumption that would occur if there were no mass transport limitations inside the matrix, i.e., if the concentration is the same everywhere in the biofilm.

Apart from the diffusional limitation inside the biological pellicle, a mass transfer limitation can also take place outside the biofilm, where the substrate must be transported from the bulk fluid to the surface of the biofilm, through a stagnant layer. The flux of substrate across the stagnant layer is equal to the substrate consumption inside the microbial film, and can be expressed in terms of an external mass transport coefficient, $k_{\mathrm{m}}$, calculated from empirical correlation:

$r_{\mathrm{fa}}=k_{\mathrm{m}}\left(C_{\mathrm{b}}-C_{\mathrm{s}}\right)$,

where $C_{\mathrm{s}}$ is the concentration of substrate at the biofilm surface and $C_{\mathrm{b}}$ is the bulk substrate concentration. In the case of first-order reaction, $r_{\mathrm{fa}}$ is expressed by:

$r_{\mathrm{fa}}=\mathrm{k}_{1 \mathrm{f}} C_{\mathrm{s}} \eta L_{\mathrm{f}}$.

If the concentration at the interface, $C_{\mathrm{s}}$ is replaced by Eq. (10), the substrate consumption by the microbial film will be given by:

$r_{\mathrm{fa}}=\frac{C_{\mathrm{b}}}{\frac{1}{k_{\mathrm{m}}}+\frac{1}{\eta \mathrm{k}_{1 \mathrm{f}} L_{\mathrm{f}}}}$,

where $C_{\mathrm{b}}$ is the bulk substrate concentration and $L_{\mathrm{f}}$ the biofilm thickness.

The mass transport phenomena inside the microbial film can be expressed by a mass transfer coefficient in the biofilm, if the following relation is used:

$k_{\mathrm{b}}=\frac{D_{\mathrm{f}}}{L_{\mathrm{f}}}$,

where $k_{\mathrm{b}}$ is the average mass transfer coefficient over the depth of the biofilm. Thus, the Thiele modulus (equation 7) can be expressed by:

$\phi=\sqrt{\frac{\mathrm{k}_{1 \mathrm{f}} L_{\mathrm{f}}}{k_{\mathrm{b}}}}$.

The $k_{\mathrm{b}}$ values were experimentally determined for a non-consumed substance, the lithium ion. The respective values for the substrate were determined assuming that:

$k_{\mathrm{b}_{\text {glicose }}}=k_{\mathrm{b}_{\mathrm{Li}^{+}}} \frac{\text { Equivalent diameter of the substrate molecule }}{\text { Diameter of the } \mathrm{Li}^{+} \text {ion }}$.

Rearranging Eq. (12) the consumption rate of the limiting substrate by the biofilm, $r_{\text {fa }}$, can be expressed by the following equation:

$r_{\mathrm{fa}}=\frac{C_{\mathrm{b}}}{\frac{1}{k_{\mathrm{m}}}+\frac{1}{\left(\tan h \sqrt{\frac{\mathrm{k}_{1 \mathrm{f}} L_{\mathrm{f}}}{k_{\mathrm{b}}}}\right) \sqrt{\mathrm{k}_{1 \mathrm{f}} L_{\mathrm{f}}} \sqrt{k_{\mathrm{b}}}}}$.

This equation can be resolved with respect to $\mathrm{k}_{1 \mathrm{f}} L_{\mathrm{f}}$, all the other variables being known.

By analogy with the "specific growth rate" of microorganisms, a new variable called "biofilm specific production rate", $\mathrm{kg}_{\text {biofilm produced }} / \mathrm{kg}_{\text {biofilm on the surface }} \mathrm{s}$, can be defined as:

$\mu_{\mathrm{p}}=\frac{J_{\mathrm{p}}}{m_{\mathrm{f}}}$,

and related to process variables and biofilm properties by:

$\mu_{\mathrm{p}}=\frac{Y_{\mathrm{X} / \mathrm{s}} r_{\mathrm{fa}}}{\rho_{\mathrm{f}} L_{\mathrm{f}}}$,

or, expressing $r_{\mathrm{fa}}$ by the corresponding equation in terms of biofilm efficiency, the surface concentration, the film thickness and the first order rate constant [6]: 
$\mu_{\mathrm{p}}=\frac{Y_{\mathrm{X} / \mathrm{s}} \mathrm{k}_{1 \mathrm{f}} C_{\mathrm{s}} \eta L_{\mathrm{f}}}{\rho_{\mathrm{f}} L_{\mathrm{f}}}$,

where $\rho_{\mathrm{f}}$ is the "dry" density of the biofilm (dry mass per unit volume).

\section{3}

\section{Materials and methods}

The model was applied to the data obtained in an experimental set-up briefly described as follows (for more details, see [16]): The microorganism used as a biofilm producer was Pseudomonas fluorescens grown aerobically in a continuous culture at $27^{\circ} \mathrm{C}$ and $\mathrm{pH} 7$, using glucose as the limiting substrate. The growth medium used was composed of glucose $(0.5 \%)$, peptone $(0.25 \%)$ and yeast extract $(0.125 \%)$ in distilled water, sterilized at $120^{\circ} \mathrm{C}$. The bacteria were continuously fed to a 12 liter tank, together with water and nutrients. The dilution rate in the tank was $1.2 \mathrm{~h}^{-1}$, high enough to prevent planktonic growth, the glucose concentration $20 \mathrm{mg} / \mathrm{l}$ and bacterial concentration $6 \times 10^{7}$ cells $/ \mathrm{ml}$. This "test fluid" was pumped to the test cells where biofilms were formed at different fluid velocities, ranging from 0.28 to $1 \mathrm{~m} / \mathrm{s}$. Glucose concentrations were determined colorimetrically using a Sigma Diagnostics Enzimatic Glucose Determination Kit.

Biofilm accumulation was monitored by measuring the heat transfer resistance of the biological deposit as described in the previous paper [9], which was transformed in mass units. Mass transfer coefficient determined for each biofilm during its formation, with non invasive methods [16]. Both methods are based on the fact that the biological pellicle introduces additional resistance to heat and to mass transfer, that change according to the structure or/and to the amount of biofilm accumulated.

Mass transfer measurements were carried out on a daily basis in a test section of geometry identical to the heat transfer test section. It was made of perspex, semi-circular, and positioned vertically and it included a test cell of $15 \mathrm{~cm}$ long and hydraulic diameter $2.06 \mathrm{~cm}$, where the mass transfer measurements were carried out. It had two compartments separated by a $10 \mathrm{~cm}$ long membrane of $0.21 \times 10^{-2} \mathrm{~m}^{2}$ area, $0.22 \mu \mathrm{m}$ pore diameter, impermeable to the bacteria but not to solutes. The "test fluid" flew over the membrane where the biofilm was growing. Water circulated in the other compartment [16]

The mass transfer rate in the biofilm was studied using lithium chloride, an inert substance not consumed by bacteria. This procedure was carried out without biofilm ("clean membrane"), and at different stages of biofilm formation, for biofilms formed under different fluid velocities. Basically, the mass transfer resistance offered by the biofilm is equal to the difference between the overall mass transfer resistances in the system with and without the biofilm attached to the membrane.

\section{4}

\section{Experimental results}

The model was applied to experimental data of steadystate and non-steady biofilm accumulation. Experimental values of mass transfer coefficients inside the biofilm, as a function of velocity and time were introduced in the model.

The overall substrate consumption rate was calculated from the values of the biofilm production rate presented in Table 1 as a function of velocity. The growth yield $\left(Y_{\mathrm{X} / \mathrm{S}}\right)$ for Pseudomonas fluorescens inside the biofilm was assumed to be the same as the related value in suspension and equal to $0.93 \mathrm{~kg}$ biomass $/ \mathrm{kg}_{\text {substrate }}$ [17]. The values of the external mass transfer coefficient, calculated through an empirical correlation [17] and the substrate surface concentrations calculated through Eq. (9), are also presented in Table 1 .

The glucose mass transfer coefficients, calculated from the respective lithium values [16], using Eq. (13) are listed in Table 2.

\section{5}

Application of the model to steady-state biofilms

The effectiveness factor and the average biofilm specific growth rate for the steady-state biofilms formed at different velocities, calculated, according to the Eqs. (8) and (18) respectively, are presented in Table 3 . The dimensionless concentration profiles are plotted in Figure 1.

\section{6}

Application of the model to non steady-state biofilms

The effectiveness factors for several biofilms as a function of the velocity and time are presented in Table 4.

Figure 2 shows the calculated average specific biofilm production rates, $\mu_{\mathrm{p}}$, for the different Reynolds numbers, as a function of time.

Table 1. Experimental and calculated values used on the model application

\begin{tabular}{|c|c|c|c|}
\hline \multicolumn{2}{|c|}{ Experimental Data } & \multicolumn{2}{|c|}{ Calculated values } \\
\hline $\mathrm{Re}$ & $\stackrel{J_{\mathrm{p}}}{\left(\mathrm{kg}_{\text {biofilm }} / \mathrm{m}^{2} \mathrm{~s}\right)}$ & $\mathrm{k}_{\mathrm{m}}(\mathrm{m} / \mathrm{s})$ & $C_{\mathrm{s}} \mathrm{kg} / \mathrm{m}^{3}$ \\
\hline 6695 & $9.09 \times 10^{-8}$ & $8.66 \times 10^{-6}$ & $8.69 \times 10^{-3}$ \\
\hline 8250 & $7.63 \times 10^{-8}$ & $1.02 \times 10^{-5}$ & $1.20 \times 10^{-2}$ \\
\hline 11000 & $6.24 \times 10^{-8}$ & $1.29 \times 10^{-5}$ & $1.48 \times 10^{-2}$ \\
\hline 14700 & $5.09 \times 10^{-8}$ & $1.62 \times 10^{-5}$ & $1.66 \times 10^{-2}$ \\
\hline
\end{tabular}

Table 2. Values of the glucose mass transfer coefficients inside biofilms formed at different Reynolds numbers

\begin{tabular}{lllll}
\hline $\begin{array}{l}\text { Time } \\
\text { (days) }\end{array}$ & \multicolumn{4}{l}{ Biofilm mass transfer coefficient, $k_{\mathrm{b}}$} \\
\cline { 2 - 5 } & $\mathrm{Re}=6695$ & $\mathrm{Re}=8250$ & $\mathrm{Re}=11000$ & $\mathrm{Re}=14700$ \\
\hline 1 & $2.40 \times 10^{-6}$ & $3.97 \times 10^{-6}$ & $3.22 \times 10^{-6}$ & $5.22 \times 10^{-6}$ \\
2 & $1.41 \times 10^{-6}$ & $3.01 \times 10^{-6}$ & $5.00 \times 10^{-6}$ & - \\
3 & $3.21 \times 10^{-6}$ & $2.55 \times 10^{-6}$ & $4.36 \times 10^{-6}$ & $2.81 \times 10^{-6}$ \\
4 & $1.90 \times 10^{-6}$ & - & $3.06 \times 10^{-6}$ & $1.89 \times 10^{-6}$ \\
5 & - & $1.54 \times 10^{-6}$ & $2.47 \times 10^{-6}$ & $1.80 \times 10^{-6}$ \\
6 & $1.84 \times 10^{-6}$ & $1.72 \times 10^{-6}$ & $1.87 \times 10^{-6}$ & $2.12 \times 10^{-6}$ \\
7 & $2.56 \times 10^{-6}$ & - & - & - \\
Steady- & $1.66 \times 10^{-6}$ & $1.60 \times 10^{-6}$ & $1.87 \times 10^{-6}$ & $1.85 \times 10^{-6}$ \\
state & & & & \\
\hline
\end{tabular}




\section{7}

\section{Discussion}

\section{1}

\section{Steady-state}

For steady-state conditions, the effectiveness factor of the biofilm increases (the Thiele modulus decreases) as the velocity of the water in contact with the biofilm increases (Table 3). The thinner biofilms, formed at higher velocities (previous work carried out by Vieira et al. [16] in the same experimental set-up has shown that the steady-state thickness decreases with an increase in the velocity) are completely penetrated by the substrate, contrary to the films formed under lower velocities (Fig. 1). In fact, this figure shows that depletion of substrate inside the biofilm occurs for the lowest velocity $(\operatorname{Re}=6695)$. Therefore, whether there is or not complete substrate penetration in the biofilm, the thickness of the active layer (layer of biofilm that is actively metabolising substrate) depends on the velocity of the fluid under which the biofilm was formed.

The analysis of Fig. 1 shows that, even if the biofilms formed at lower velocities are not completely penetrated by the substrate, the concentration profile is sharper than in the case of biofilms formed at higher velocities. According to van Loodsdrecht et al. [15], the outgrowth of a biofilm will be a function of the transport rate of the limiting substrate, the biomass yield and/or the biomass

Table 3. Steady-state effectiveness factors and average biofilm specific growth rate for biofilms formed under different fluid velocities

\begin{tabular}{lll}
\hline $\operatorname{Re}$ & $\begin{array}{l}\text { Effectiveness } \\
\text { factor, } \eta\end{array}$ & $\begin{array}{l}\text { Average biofilm } \\
\text { specific growth rate, } \\
\mu_{\mathrm{p}}, \mathrm{s}^{-1}\end{array}$ \\
\hline 6695 & 0.15 & $5.87 \times 10^{-6}$ \\
8250 & 0.23 & $5.55 \times 10^{-5}$ \\
11000 & 0.40 & $5.12 \times 10^{-5}$ \\
14700 & 0.52 & $5.33 \times 10^{-5}$ \\
\hline
\end{tabular}

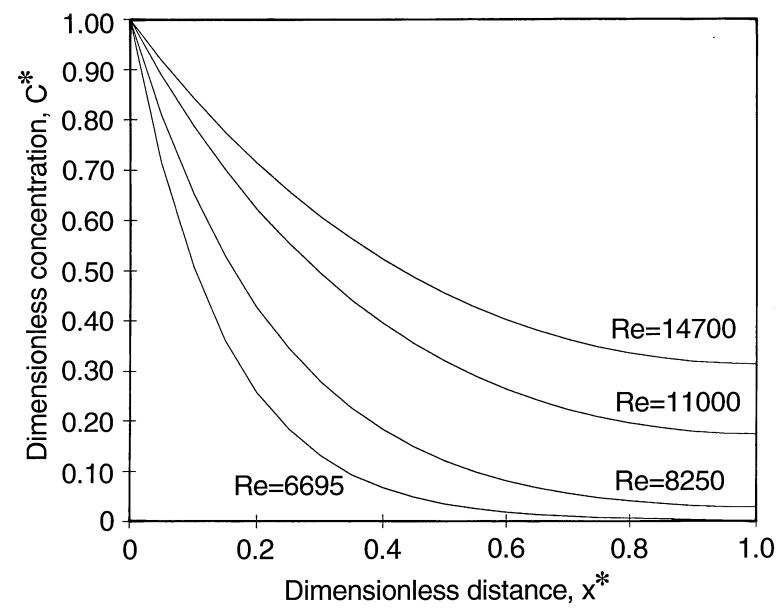

Fig. 1. Calculated steady-state concentration profiles of the limiting substrate inside biofilms formed under different hydrodynamic conditions density in the biofilm. Thus, the concentration reduction throughout the thickness of the biofilm is due not only to mass transfer limitations but also to the consumption by the microorganisms. It is probable that the cell concentration, or their activity, in the active layers of the less compact (thicker) biofilms is higher, enhancing the substrate consumption inside the biofilm. Biofilms formed at lower velocities are less susceptible to shear forces, growing faster and forming a more open structure, where the biomass concentration may be higher. In fact, several authors $[5,15]$ suggested that the microorganisms inside the biofilm "sense" a higher shear stress, producing a stronger matrix to protect themselves. Probably this effect is obtained by altering the microbial processes within the biofilm, including the synthesis of the extracellular polymers [5]. For that reason, the ratio EPS produced/cells produced may increase with water velocity.

Additionally, as the velocity decreases, the roughness of the biofilm increases and filaments can appear: as the actual biofilm area is not equal to the horizontally projected area, the amount of biofilm containing active cells may be higher than in the case of a flat surface. Associated with these conditions, the consumption of substrate is higher in the layers next to the surface of the biofilm, and depletion or low concentrations of substrate may occur at some

Table 4. Effectiveness factors for biofilms as a function of velocity and time

\begin{tabular}{lllll}
\hline $\begin{array}{l}\text { Time } \\
\text { (days) }\end{array}$ & $\operatorname{Re}=6695$ & $\mathrm{Re}=8250$ & $\mathrm{Re}=11000$ & $\mathrm{Re}=14700$ \\
& $\eta$ & $\eta$ & $\eta$ & $\eta$ \\
\hline 0 & 1 & 1 & 1 & 1 \\
1 & 0.21 & 0.53 & 0.60 & 0.81 \\
2 & 0.12 & 0.43 & 0.73 & 0.75 \\
3 & 0.28 & 0.37 & 0.69 & 0.66 \\
4 & 0.22 & 0.22 & 0.58 & 0.53 \\
5 & - & 0.23 & 0.51 & 0.51 \\
6 & - & 0.25 & 0.40 & - \\
7 & 0.23 & - & - & - \\
\hline
\end{tabular}

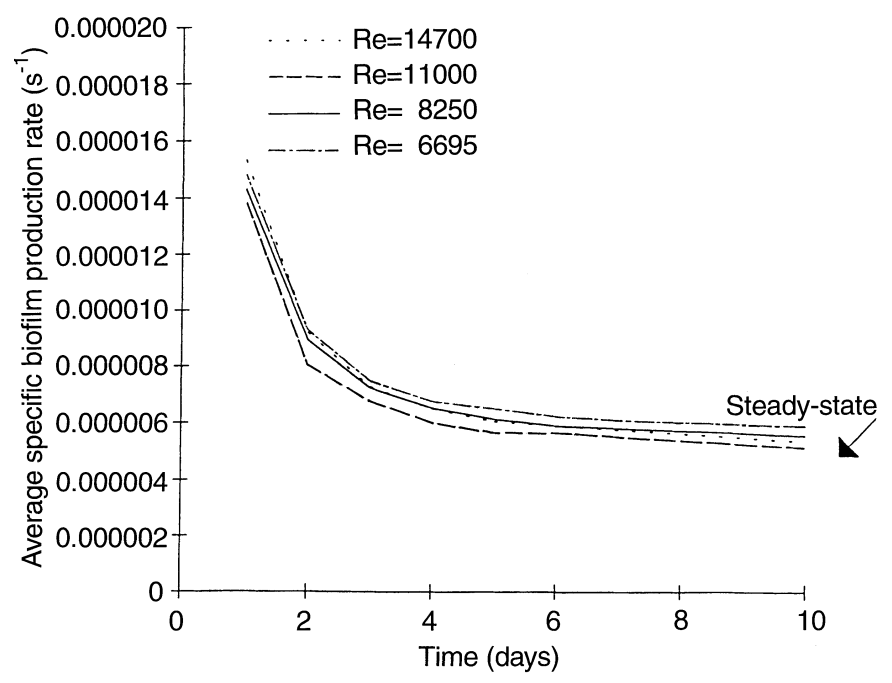

Fig. 2. Average specific biofilm production rate $\left(\mathrm{s}^{-1}\right)$ as a function of time 
point within the biofilm, reduction or stopping the reaction from that point on. Based on this, it is not surprising that these biofilms have a lower effectiveness factor than biofilms completely penetrated by the substrate, even if the former consume more substrate per unit area and time.

Although it was not possible to experimentally confirm this hypothesis, substrate consumption per unit area and time was calculated $\left(r_{\mathrm{fa}}\right)$ by Eq. (2) and is presented in a previous paper [9]. This value decreases from $9.8 \times 10^{-8}$ to $5.5 \times 10^{-8} \mathrm{~kg} / \mathrm{m}^{2} \mathrm{~s}$, as the Reynolds number increases from 6695 to 14700 .

The average biofilm specific production rate (per unit mass of biofilm) in steady-state seems to be independent of the fluid velocity under which the biofilm is formed (Table 3). These very similar values were not expected, since biofilms completely penetrated by the substrate should have higher biomass production rate per unit of biofilm mass. Nevertheless, the velocity has two contradictory effects on biofilm formation: on one hand, biofilms formed under higher velocities are thinner, giving raise to completely penetrated films (all the biofilm is active and, consequently, tends to be more efficient); on the other hand, these biofilms are more dense and compact [15] reducing the benefit of low thickness on mass transfer. This last statement is also supported by the identical mass transfer values obtained, at steady-state, for every biofilm formed (Table 2).

Contrary to the results presented in Table 2, Nagaoka [10] investigating mass transfer coefficients in biofilms under oscillatory flow conditions, suggested that substrate uptake rate by biofilms increased with the increase of the Reynolds number of the wave motion. However, the experimental method used by this author - a biofilm was grown on a plate and the substrate uptake rate by the biofilm was measured changing the hydraulic condition of the channel for a short period to investigate the short term effect of the change of the bulk condition - does not allow enough time to originate changes in the internal structure of the matrix caused by the velocity. In that case, the conclusion was that the substrate transport in those biofilms is carried out by turbulent diffusion. Brito [2] has also reported similar results, i.e., an enhancement of the substrate removal due to an increase of the liquid velocity, but these results were also justified by a reduction of the substrate external mass transfer resistance, that was controlling the reaction, and not by the fact that the hydrodynamic conditions can affect the biofilm behaviour.

\section{2}

\section{Unsteady-state}

From the analysis of Table 4, it can be concluded that, in every case, the biofilm effectiveness factor decreases with time, as the amount of biofilm accumulated on the surface increases. This effect is more pronounced for biofilms formed at lower velocities. In the end, the effectiveness factor of the biofilm tends to a constant value that is higher for higher shear rates.

Furthermore, Fig. 2 shows that for all the velocities studied the average specific biofilm production rate is very high in the initial phase of biofilm formation, decreasing as the amount of biofilm on the surface increases. Initially, the biomass that constitutes the biofilm is basically cells, which means that almost all biomass may produce new cells and extracellular polymeric substances (EPS). After this initial phase, only a fraction of the mass of the biofilm (cells) is able to produce new products and cells, which means that the ratio "biomass produced/biomass on the surface" decreases with time. Moreover, as the thickness increases, the substrate concentration inside the biofilm decreases and a new microbial distribution inside the matrix may occur. As the growth is a first order reaction with respect to substrate concentration, it decreases within the biofilm as the distance from the interface liquid-biofilm increases, affecting also the average value of the biofilm production rate.

\section{8}

\section{Conclusions}

In this paper a first order diffusion-reaction model was applied to experimental results of biofilm accumulation by a pure culture of Pseudomonas fluorescens, under turbulent flow and low substrate loading rate, using data corresponding to the non-steady-state and steady-state conditions. The substrate consumption rate of the biofilm was calculated based on the on-line determination of the amount of biofilm accumulated on the surface. It must be stressed that this approach is important when the residence time or the substrate concentration in the reactor is very low, and concentration measurements and mass balances cannot determine the substrate consumption rate. Also, instead of assuming values for the internal diffusivity of the substrate, values of the internal mass transfer coefficient were measured and introduced in the model.

For steady-state conditions the rate of substrate consumption decreases with increasing velocity, meaning that changes in hydrodynamic conditions in turbulent flow have a significant effect on biofilm behaviour. The fraction of biofilm penetrated by the substrate depends also on the velocity of the fluid that contacts the film: contrary to the biofilms formed at lower velocities, the pellicles formed at higher velocities are completely penetrated by the substrate. This fact is associated with the biofilm structure in terms of biomass density and the compactness of the matrix, due to the shear stress exerted on the biofilm by the fluid flowing over it. During the unsteady-state period, biofilms formed at higher velocities have higher effectiveness factors, and the biofilm production rate is very high in the initial phases of biofilm formation, decreasing both as it becomes thicker.

In conclusion, biofilms formed under high shear stresses are thinner and denser, and are completely penetrated by the substrate. Although they remove less substrate per unit volume of reactor, they are more stable. In fact, biofilms formed at lower velocities are more susceptible to sloughing off, due to the probable presence of inactive layers. In addition, these biofilms are less compact and possess protuberances, which can easily cause detachment from the surface. Moreover, these biofilms are less resistant to slight changes in the hydrodynamic conditions [16], that often occur in bioreactor systems.

These results are in accordance with those presented by van Loosdrecht et al. [15] who managed to control biofilm 
growth, and stabilising the reactor system, by the application of high shear stresses. Conversely, there are some reactors (as in the case of biofilm fluidised bed reactors) where high shear stresses cannot be achieved and the biofilm continues to grow, affecting reactor stability. So, better reactor operation is expected to occur for the conditions of thinner biofilms.

The conclusions obtained so far can be used to predict biofilm behaviour in some circumstances. Concerning biocide action to control biofilm growth (particularly important in the case of unwanted biofilms, namely on heat exchanger surfaces and drinking water networks) it is obvious that the biocide should be able to penetrate all the biofilm in order to interact with its target, i.e., the bacterial cell. So, it is expected that biofilms formed at higher velocities (and completely penetrated by the substrate) should be more easily penetrated by the substance chosen to control biofilm growth. Additionally, as biocides are expected to react with bacterial cells, interacting with their metabolism, they will probably be more effective when the cell in the biofilm is metabolically active.

As regards the ability of the biofilm to recover after sloughing off, it is expected that it will be easier to happen if the biofilm is completely penetrated by the substrate (i.e., biofilms formed at higher velocities) due to the fact that all the bacteria, specially those that are in the lower layers of the pellicle (the "survivors") are still active and capable of producing new cells. Besides that, if the biofilm is pure (produced from a known inoculum), probably the occurrence of sites of depletion of substrates (in the case of thicker biofilms) may favour the growth of contaminants (e.g., anaerobic zones in aerobic biofilms).

\section{References}

1. Applegate D.H.; Bryers, J.D.: Effects of Carbon and Oxygen Limitations and Calcium Concentrations on Biofilm Removal Processes. Biotechnol. Bioeng. 37 (1991) 17-25

2. Brito, A.: Low Strength Waste Water Treatment with UASB/ EGSB and SBR reactors - Biological Kinetics and Mass Transfer, $P h D$ thesis (in portuguese), Univ. Minho, 1995

3. Cao, Y.S.; Alaerts, G.J.: Influence of Reactor Type and Shear Stress on Aerobic Biofilm Morphology, Population and Kinetics. Wat. Res. 29 (1995) 107-118

4. Coelhoso, I.; Rodrigues, A.: Modeling of Biofilm Reactors with consecutive Reactions. Biop. Eng., 12 (1985) 187-192

5. Fletcher, M.: "Bacterial Metabolism in Biofilms" In Melo, Bott, Fletcher and capdeville (Eds): Biofilms-Science and Technology, 113-124. Dordrecht, The Netherlands: Kluwer Academic Publishers 1992

6. Harremöes, P.: "Biofilm Kinetics" In: Mitchell, R.(Ed.): Water Pollution Microbiology, vol 2, pp 82-109. New York: John Wiley and Sons 1978

7. Lewandowski, Z.; Stoodley, P.; M Altobelli, S.: Experimental and Conceptual Studies on Mass Transport on Biofilms. Wat. Sci. Tech. 31 (1995) 153-162

8. Libick, S.B.; Salmon, P.M.; Robertson, C.R.: The Effective Diffusive Permeability of a non reacting Solute in Microbial Cell Aggregates" Biotechnol. Bioeng. 32 (1988) 68-85

9. Melo, L.F.; Vieira, M.J.: Physical Stability and Biological Activity of Biofilms Formed Under Turbulent Flow And Low Substrate Concentration, Submitted.

10. Nagaoka, H.: Mass Transfer Mechanisms in Biofilms under oscillatory conditions. Wat. Sci. Tech. 36 (1997) 329-336

11. Onuma, M.; Omura, T.: "Mass Transfer Characteristics within Microbial Systems" Wat. Sci. Tech. 14 (1982) 553-568

12. Peyton, B.M.: Effects of Shear Stress and Substrate Loading Rate on Pseudomonas aeruginosa Biofilm Thickness and Density. Wat. Res. 30 (1996) 29-36

13. Rittman, B.E.; McCarty, P.L. "Model of steady-State-Biofilm Kinetics" Biotechnol.Bioeng. 22 (1980) 2343-2357

14. Stoodley, P.; de Beer, D.; Lewandowski, Z.: Liquid Flow in Biofilm Systems. Appl. Environ. Microbiol. 60 (1994) 27112716

15. Van Loosdrecht, M.C.M.; Eikelboom, D.; Gjaltema, A.; Mulder, A.; Tijhuis, L.; Heijnen, J.J.: Biofilm structures. Wat.Sci.Tech. 32 No 8 (1995) 35-43

16. Vieira, M.J.; Melo, L.M.; Pinheiro, M.M.: Biofilm formation: Hydrodynamoc Effects on Internal Diffusion and Structure, Biofouling, 7 (1993) 67-80

17. Vieira, M.J.: Study of the formation of biological films by Pseudomonas fluorescens and of the effects associated to internal mass transfer and incorporation of kaolin particles, $P h D$ thesis (in portuguese), Univ Minho, 1995

18. Zhang, T.C.; Bishop, P.L.: Density, Porosity and Pore structure of Bioflims. Wat. Res. 28 (1994) 2267-2277 